\title{
LEGITIMIDAD DEL ESTADO COLOMBIANO: UNA APROXIMACIÓN A LOS GONGEPTOS DE GOBERNABILIDAD Y GOBERNANZA'
}

(Recibido: Julio 3 de 2012 Aprobado: Agosto 14 de 2012)

Diego Edgardo Rojas Escobar ${ }^{2}$

\begin{abstract}
Los individuos se relacionan con el gobierno y acceden a los servicios del Estado y a un desarrollo así sea de modo precario y desigual. (Fernán González, 1997)
\end{abstract}

\section{Resumen}

En el presente artículo se realiza un acercamiento a la realidad colombiana a partir de las expresiones de legitimidad que el Estado y la sociedad misma, pueden manifestar al contexto que les es propio, aunque las situaciones sean diferentes. A partir de esto, se toma como eje principal los conceptos de Gobernabilidad y Gobernanza, que permiten vislumbrar la relación que puede llegar a existir entre gobernantes y gobernados en un escenario donde se busca ampliar el diálogo entre ambas partes y, así mismo, disminuir las demandas sociales.

De este modo y tenida como referente fundamental la Constitución de 1991, en donde se busca un mayor reconocimiento de los derechos humanos y la participación ciudadana, se plantea la idea de una sociedad activa, existente, que exige a diario mejores condiciones de vida y mayores niveles de igualdad, aspectos que deben ser garantes de un Estado enfocado a ampliar los niveles de legitimidad en su territorio.

\section{Palaburas clave}

Legitimidad, Gobernabilidad, Gobernanza, Conflicto, Constitución.

\section{IEGITIMACY OF THE COLOMBIAN STATE: AN APPROACH TO THE CONCEPTS OF GOVERNABILITY AND GOVERNANGE}

\section{Alostract}

This research article makes an approach to the Colombian reality from the state and the society's legitimacy. It makes part of the social and cultural issues; it is opened to history and to particular changes. As Launay and Gonzalez $(2010,25)$ would say: "legitimacy talks about everything that inspires the obedience of a political actor". In this sense, terms such as governance and government procedure are taken as main axes that will allow understanding the probable relationship between leaders and governed people in a scenario where they try to widen the dialogue between both parties so as to decrease the demands presented in a society.

So, considering the 1991 Constitution as a fundamental point of reference that looks for a greater recognition of human rights and the citizen's participation, the idea of having an active and existing society is considered. It requires things such as better life conditions and higher levels of equality, which should guarantee a state focused on widening the levels of legitimacy in its territory.

\section{Kev words}

Legitimacy, governance, government procedure, conflict, constitution.

1 Artículo de reflexión, producto parcial del proyecto "Imaginario Político de los jóvenes de la Universidad Santo Tomás, Tunja" en la línea de Hombre, Sociedad y Ética del grupo de Investigación Expedicionarios Humanistas, perteneciente al Departamento de Humanidades de la Universidad Santo Tomás, Tunja.

2 Docente del Departamento de Humanidades de la Universidad Santo Tomás, Tunja. Licenciado en Filosofía, Ética y Valores Humanos, Universidad Santo Tomás. Especialista en Gobierno, Gerencia y Asuntos Públicos, Universidad Externado de Colombia. Coordinador de área de Filosofía Política. E-mail: drojas@ustatunja.edu.co 


\section{Introducción}

Algunos conceptos de gran interés en este artículo son el de 'gobernabilidad' y 'gobernanza', ya que se han convertido en un soporte fundamental $y$, por qué no, en el eje principal de la legitimidad en un Estado, pues, a lo largo de los últimos años estos conceptos se han transformado en el punto central de aquellas políticas encaminadas a generar grandes niveles de desarrollo y alianzas interestatales, esto con el propósito de que las políticas propuestas sean una realidad, que se vive no sólo en las estructuras estatales, sino en las estructuras sociales, ya que estas últimas, son agentes activos en el proceso de una verdadera gestión pública.

De otro lado, hay que dejar en claro que conceptos como 'gobernabilidad' y 'gobernanza' hacen referencia a dinámicas políticas distintas y tienen definiciones propias, pero de igual forma en muchos aspectos, tienden a ser confundidas. De acuerdo con el diccionario de la real academia 'gobernabilidad' es la cualidad de gobernable y 'gobernanza' es el arte o la manera de gobernar que se propone como objetivo el logro de un desarrollo económico, social e institucional duradero promoviendo un sano equilibrio entre el Estado, la sociedad civil y el mercado de la economía.

Desde el campo de las ciencias sociales, podemos citar a Jan Koiman (1993), quien define la gobernabilidad de la siguiente manera "la gobernabilidad es la capacidad de un sistema sociopolítico para gobernarse a sí mismo en el contexto de otros sistemas más amplios de los que forma parte" (Koiman, 1993, 32).

es decir, se debe tener presente aqueIla capacidad que debe existir dentro de un Estado o sistema político para que, cada vez, sea más estable, a partir de sus políticas propuestas, como generadora de resultados concretos, acertados y sostenibles, en sí, se refiere a ciertas condiciones que asumen los sistemas sociopolíticos para gobernar.

De acuerdo con las ciencias sociales, se hace pertinente abordar y definir el concepto de gobernanza, donde éste se refiere no a la capacidad, sino a un proceso o a un conjunto de interacciones sociopolíticas y sus específicos resultados (Launay y González, 2010, 14), propuesta que permite tener presente que el proceso de la gobernanza se hace dinámicamente, donde el puente que sostiene dicho proceso se llama interacción, constante diálogo entre lo que se piensa, se quiere y se necesita, para la obtención de resultados concretos y acertados.

Se entiende, entonces, que la preocupación por la gobernabilidad remite a las condiciones que hacen gobernable y eficiente la administración de una determinada sociedad y, por esa vía, a la evaluación de las estructuras y del funcionamiento de las instituciones. En cambio, el enfoque de la gobernanza se refiere a un conjunto de procesos, regulaciones e interacciones políticas que harían posible un "desarrollo (...) duradero" y un "sano equilibrio" entre los actores que conforman una sociedad (Launay y González, $2010,14)$.

Un tema de gran preocupación es el de saber si en realidad en el contexto colombiano se goza de niveles de gobernabilidad y gobernanza que dejan como resultado un Estado que refleja constantemente características de legitimidad, pues, una particularidad de estos dos conceptos fundamentales es el de tener presente que cuando un Estado se sostiene sobre principios bien estructurados, permite poder dar respuesta de manera eficiente a las demandas de la sociedad civil.

A partir de esto se puede ver como resultado un tercer concepto, el de buena gobernabilidad, que es entendido de la siguiente manera: una buena gobernabi- 
lidad se da cuando existe una respuesta gubernamental eficiente frente a los problemas de una sociedad. Por el contrario, una situación de ingobernabilidad se expresaría en la "desinstitucionalización" de las instancias de la representación política -tales como los partidos y el Congresoy en la debilidad y desorganización del aparato estatal (Ungar, 1997, 13).

Esto permite dejar en claro que es el Estado el destinatario primordial de las peticiones de la sociedad civil $y$, aunque suene duro, hay que decirlo con madurez, el Estado colombiano ha sido identificado por tener un déficit de gobernabilidad, ya que ha sido un Estado, por decirlo así, impotente, es decir, un Estado que no ha podido tener el dominio en la totalidad de su territorio, que ha permitido el monopolio de la violencia legítima. En la historia del territorio colombiano, se ha podido ver claramente que han existido lugares que están fuera del control del Estado y otros lugares donde se asigna el control del mismo a los llamados poderes locales. Realidad que ha permitido ver la relación existente entre las instituciones pertenecientes al Estado con el pueblo y el territorio con el término de presencia diferenciada del Estado (González, Bolívar y Vázquez, 2003).

Esta presencia diferenciada da cuenta de la manera como el funcionamiento y la organización de las agencias estatales son diferentes en la regiones más integradas del país, en las regiones marginales y en las regiones en proceso de articulación al conjunto de la nación. Esta diferenciación ha sido caracterizada a veces en términos dualistas, desde la contraposición entre modernidad y tradición, que se expresa en la coexistencia de instituciones políticas y económicas de carácter "moderno" e "impersonal", e instituciones consideradas "tradicionales", basadas en relaciones clientelistas y solidaridades personalizadas "cara a cara". Esta coexistencia explica la importancia de la intermediación que los partidos tradicionales y los actores armados han jugado entre las instituciones estatales y los poderes de facto existentes en regiones y localidades (Launay y González, 2010, 31).

De ahí que en diferentes textos o diversos autores, se pueda leer en diversas ocasiones que en Colombia la política no está plenamente estatalizada. Esto significa que muchos actores que no hacen parte del Estado tienen responsabilidades relevantes como reguladores políticos y que muchas de las regulaciones ejercidas no se ajustan a los códigos estatales, es decir, a la ley. Es por esto, que existen diversas formas de gobernanza según el nivel y el tipo de presencia del Estado en las diferentes partes de su territorio. A partir de esto, la propuesta que ahora se plantea es la de poder comprender la gobernanza y la gobernabilidad como prácticas que se encargan de regular la política, para garantizar niveles de legitimidad estatal.

\section{Gobernantes + Goberna-Dos = ¿Gobernanza?}

Uno de los principios fundamentales en este análisis que ahora se hace, es el de saber que una gobernanza se convierte en algo legal cuando la actividad del poder respeta aquel conjunto de reglas y principios, que pasan a ser respaldados por la ley. En cambio la noción de legitimidad es mucho más abierta a la historia y a la variación local, en efecto, la legitimidad habla de aquello que inspira la obediencia de un actor político (Launay y González, $2010,25)$, de todas formas esto permite dejar en claro que esta legitimidad se convierte en una dimensión primordial como una condición igualmente fundamental para la gobernanza.

El problema de esto, se presenta cuando no hay acuerdos comunes frente a los diferentes motivos existentes entre 
los grupos para asignar legitimidad a ciertos actos de gobierno o sistemas de poder. Es por esto, que la aplicación de las categorías gobernanza/gobernabilidad a Colombia hace necesario distinguir esas distintas legitimidades que compiten entre sí.

Como se ha mencionado en renglones anteriores, ha existido una falta de control de la totalidad del territorio por parte del Estado, pero esto no ha existido en épocas actuales, es una realidad que se ha presentado desde tiempos de la colonia, donde dejaba territorios y poblaciones enteras por fuera de la administración española, y esto es lo que ahora el Estado colombiano ha tenido que asumir, pero que no ha podido remediar.

Realidad que ha llevado a que se desaten otras situaciones que de igual manera el Estado no ha podido sobreponer, aspectos como la conformación y consolidación de movimientos guerrilleros de las FARC, ELN y EPL, quienes aprovecharon las tensiones del mundo agrario que habían sido dejados en el olvido por ese mismo Estado, situación que les permitió expandirse hacia otras zonas más ricas, otra situación por la que paso nuestro país fue aquel momento donde los grupos liberales y comunistas se organizaron en guerrillas y grupos de autodefensa campesina, mientras que de otro lado los conservadores respondían con la organización de grupos contraguerrillas, la politización conservadora de la policía (chulavitas) y el uso de grupos de asesinos (los pájaros), escenario que lleva a vivir una época de estabilidad estatal y violencia, que era permitida por el Estado, ya que éste no tenía el control de dicha realidad, debido a que le era difícil adaptarse de manera rápida a los cambios sociales.

Una situación más que no puede quedar en el olvido, es el Pacto del Frente Nacional en 1958, donde los partidos políticos tradicionales llegan a un acuerdo con el objetivo de dar fin al conflicto y a la dictadura militar, acuerdo que dejaba en claro que cada partido político se turnaría la presidencia por un periodo de 16 años y se repartirían en mitades los cargos burocráticos con el fin de civilizar la contienda bipartidista, pero este sistema no fue de gran ayuda para el pueblo, ya que era difícil articular a las regiones marginales con el Estado, lugares donde los grupos armados tomaron ventaja.

Todos estos factores, junto con los procesos de paz entre guerrillas y gobierno, abrieron el camino a la reforma constitucional de 1991. La nueva Constitución reconoció el pluralismo ético, cultural y religioso; intentó dar solución a los principales vicios del sistema político; consagró una gran variedad de derechos individuales y políticos y sentó los fundamentos de la descentralización administrativa (Launay y González, 2010, 35), esta diversidad de comportamientos políticos hace que sea más difícil asumir en la realidad colombiana los criterios de gobernabilidad y gobernanza, asumidos de modelos de desarrollo político de los países denominados, política y económicamente desarrollados.

De otro lado, se ha presentado un fenómeno denominado, "abstencionismo electoral", que para nuestros días supera el $50 \%$, debido a que la clase política y el sistema de representación carecen de legitimidad, aunque las instituciones en Colombia gozan de una amplia tradición y que el mandato de la clase política ha sido renovado asiduamente en la urnas por más de 150 años de historia electoral, casi ininterrumpida, nos permite hacer un alto, para repensar los supuestos y las pretensiones detrás del concepto de legitimidad.

Los principios básicos de las democracias modernas sostienen que los gobiernos legítimamente elegidos obtienen, por medio del consenso, el reconocimiento y la legitimidad que les permite ejercer la 
dominación sobre el territorio y sus habitantes. Por medio de la actividad electoral, las mayorías en la sociedad civil otorgan poder y autoridad a los gobernantes, que adquieren así legitimidad para gobernar. En esta óptica, un régimen político legítimo debe ser democrático (Santana, 1990) y apoyado por el consenso de la población.

Es por esto, que la legitimidad del sistema político es una condición necesaria e imprescindible para que una sociedad sea gobernable; de otra forma, las autoridades no cuentan con el poder suficiente para hacer cumplir las leyes y los mandatos en el territorio. Si el Estado es ilegítimo por no hacer cumplir las leyes y los mandatos en todo el territorio, habría que recordar entonces que ese carácter del Estado se construye de forma gradual y conflictiva mediante la articulación de regiones y sectores sociales en distintos momentos (Launay y González, 2010, 39).

Se podría afirmar entonces que un reflejo de la ilegitimidad estatal se percibe a través del clientelismo político, donde se pone en juego el imaginario colectivo de los colombianos, pues ha cogido fuerza la idea de que la política y más aún los políticos atentan contra el desarrollo social de la comunidad y afectan a la sociedad, pues son realidades que se concentran totalmente a tener un enriquecimiento individual y su permanencia en el poder. Concepción que ha tenido más fuerza por la poca representación por parte de los partidos políticos tradicionales y la poca presencia de instituciones en sectores marginados de la población.

Esta presencia diferenciada de las instituciones estatales hace que, como se ha insistido en otras oportunidades, los conceptos de legitimidad y gobernanza tengan que ver más con la forma como se gobierna, que refleja la relación existente entre gobernantes y gobernados, y no con la comparación con un modelo ideal y abstracto de democracia, tal como aparece en los manuales de ciencia política. Esa mira- da a la realidad política concreta muestra la dificultad de hablar de la viabilidad y deseabilidad de medidas modernizantes y modelos de sociedad al estilo de los parámetros de la good governance en un Estado que no ejerce el mismo control ni que tiene la misma presencia en todo el territorio; en una sociedad donde el Estado no monopoliza el ámbito de lo político; en un sistema donde conviven prácticas modernas y tradicionales. La implantación de esas reformas modernizantes encuentra muchas dificultades en ese tipo de política concreta, pues ellas presuponen la existencia de un Estado que no requiere de la mediación de otros niveles de poder para ejercer su dominación, y que goza del pleno monopolio de la violencia y de la administración de la justicia, La gobernabilidad y legitimidad que posee y proyecta un líder en un contexto similar no siempre obedece al seguimiento estricto de las formas ideales de hacer política (Launay y González, 2010, 71).

Se puede entender entonces, que la gobernabilidad y legitimidad que hace parte de un líder y que éste mismo líder propone en una realidad concreta no siempre se enfoca en el seguimiento estricto de las formas ideales de hacer política. Así, la gobernabilidad o gobernanza no es en su totalidad necesariamente el resultado del estilo de gobierno tecnocrático, meritocrático, impersonal y gerencial, que suponen los manuales, sino que puede ser producido por la combinación de diversas lógicas políticas y el cultivo de legitimidad por distintas vías.

\section{La legitimidad presente en el conflicto colombiano}

La relación entre gobernantes y gobernados, que se deduce de la concepción de gobernanza como interacción entre la sociedad y el poder existente, permite hacer un análisis más profundo acerca de la presencia diferenciada del Estado.

En el contexto colombiano, existe una situación real donde diversos actores que no son entes estatales, pueden y han 
intervenido en la vida pública: unos de ellos como las organizaciones sociales, la Iglesia católica, los sindicatos, las ONG, los movimientos de mujeres, campesinos, indígenas y afrocolombianos. Pero la situación de conflicto hace aparecer otros tipos de actores no estatales como los grupos de guerrilla, paramilitares, mafias; así como un número importante de excombatientes que ejercen en ciertos casos funciones públicas. Es a partir de estos últimos que a continuación se hará un análisis acerca de la presencia de la legitimidad en tiempos de conflicto.

Para hacer este análisis se hace pertinente hablar de algunas clases de gobernanza, una de ellas sería la gobernanza legal y otra que también nos podría ampliar más este panorama, sería la gobernanza ilegal, pues hay que tener claro aquellos momentos donde los distintos actores armados tuvieron la oportunidad de ejercer o, por qué no decirlo, ejercen un control poblacional más o menos permanente, al mismo tiempo que ejercen funciones de seguridad y abastecimiento por medio de la coerción, el interés particular o las convicciones políticas.

El accionar político, militar y económico de los actores armados ha derivado en compromisos con la dirigencia política para que sus decisiones no den tantas vueltas (un escenario gobernable) donde actores armados y clase política se vean beneficiados por las políticas públicas, pues generan legitimidad de los actores legales e ilegales, es decir, un conjunto híbrido que produzca una nueva forma de gobernanza/gobernabilidad que vaya más allá de las formas institucionales y las fórmulas preestablecidas que busca explicar las relaciones sociales y políticas en las sociedades (Launay y González, 2010, 101).

Estas relaciones de gobernabilidad, en escenarios que en cualquier otra parte del mundo pueden llegar a ser señalados como caóticos, ilustran tendencias de go- bernanza sui géneris, las cuales representan un híbrido entre legalidad e ilegalidad que sobrepasa fórmulas institucionales que plantea un deber ser de la gobernanza para regir nuestras sociedades $\sin$ distingo alguno.

Si se hace un recorrido histórico, y se analiza la realidad colombiana a partir del año 2002, podemos recordar como con el inicio de este nuevo gobierno, se inicia un proceso de desarme, desmovilización y reintegración (DDR), de combatientes de grupos armados ilegales paramilitares y guerrilleros en el país. Este proceso, que sería catalogado como una política de desarme, que está fundamentada en un discurso y una lógica de transición de un Estado en conflicto a un Estado en paz.

A su vez, requiere satisfacer las demandas de las víctimas y establecer unas políticas relacionadas con el futuro y la reinserción de excombatientes. De esa lógica, el deber ser del Estado, es abrir espacios laborales, educativos y psicosociales que permitan a los desmovilizados reintegrarse a la vida civil. El fin último de la transición es construir una sociedad pacífica, libre de violencia y prevenir el resurgimiento de conflictos armados (Rettberg, 2002 y 2005. Pauwels, 2000. Koth, 2005. Kingma, 2001). Un aspecto esencial para la construcción de paz y la transición es la desmovilización y reinserción de miembros de grupos armados al margen de la ley, pues el éxito de esos procesos depende la sostenibilidad de sociedades posbélicas.

Es por eso, que la primera institución del gobierno encargada de esta fase fue el Ministerio del Interior y de Justicia con el programa de reinserción a la vida civil (PRVC), programa que otorgaba beneficios sociales y económicos a los desmovilizados, como el acceso al sistema educativo, al sistema de salud y apoyo psicosocial, esto acompañado de una ayuda humanitaria. Pero en septiembre de 2006, se crea la alta consejería para la 
reintegración social y económica de personas y grupos alzados en armas (ACR), en donde los excombatientes dejaron de ser denominados por el gobierno nacional como reinsertados o reincorporados y pasaron a ser llamados "participantes", término que asimila la participación en un mayor compromiso individual con su proceso de reintegración. Esto permitió dejar en claro, que empieza a existir un análisis de las relaciones entre el Estado y excombatientes, en el marco de sus políticas asumidas, con el fin de aportar a la conceptualización de la gobernanza en Colombia (Launay y González, 2010, 111).

Pero hay que saber con total certeza que esto no sería la solución para garantizar el bienestar de estas personas, y lograr mayores niveles de gobernanza en Colombia, ya que, aunque es un hecho que los excombatientes reciben beneficios y apoyos económicos durante su proceso de reintegración, se puede plantear que existe un Estado que desconoce el carácter de sujetos de derechos de desmovilizados y reincorporados, al presentar como beneficios derechos fundamentales consagrados en la Constitución Política de Colombia.

Por eso, la concepción de participación que tienen algunos excombatientes diverge de la noción individualista de participación que plantea el Estado a través de sus programas de reinserción. Mientras que los excombatientes conciben la participación como una herramienta para alcanzar un lugar político, el Estado plantea la participación desde una óptica individualista y liberal (Launay y González, 2010, 114).

Estos aspectos llevan a pensar y entender que aquellas personas desmovilizadas son sujetos que se encuentran en proceso de ser parte de la civilidad dado que vienen de un orden ilegal de vuelta a un orden legal. Nos muestra de un lado, la importancia del papel de un nuevo actor no estatal considerado antes como “ilegal" de vuelta a la legalidad y de otro, las particularidades de la relación entre Estado y excombatientes en el marco del actual proceso de desarme, desmovilización y reintegración (DDR) en Colombia.

Esto deja serias inquietudes de poder construir un orden social después del conflicto en nuestro país, pero un orden que en realidad responda a las ideas de la gobernanza como interacción entre ciudadanos y Estado. Por esto, se hace necesario hacer un análisis sobre el sentido y significados de las propuestas planteadas en la Constitución del 91, donde la propuesta en marcha es aumentar los niveles de gobernanza en Colombia.

\section{La nueva propuesta de Estado a partir de la Constitución de 1991}

Como se ha podido percibir, la Gobernanza en el territorio colombiano ha tenido una característica relevante, la de una presencia diferenciada de las instituciones estatales $y$, a partir de esto se hace pertinente, o por qué no decirlo, necesario crear una política que responda a esta realidad social. Es por esto que en éste último momento se hace una lectura a las propuestas planteadas con la constitución del 91, como medio para la recuperación de la legitimidad del Estado, donde la gobernanza es vista a partir de la interacción continua entre Estado y ciudadanos.

Acorde con esto, la Constitución planteada en 1991 resulta de las deliberaciones presentes en la Asamblea Nacional Constituyente, que pretendía poner fin a la crisis política existente en aquella época, cuando la proyección de nuestro país se encontraba de frente contra el narcotráfico y la violencia política, a lo que el Estado denominó como narcoterrorismo. En tal sentido, la nueva propuesta de esta Constitución buscaba organizar la realidad política, en donde se pudiera establecer una verdadera relación entre Estado y sociedad, esto con el objetivo de 
velar y respetar los derechos propios del ciudadano para, de esta manera, empezar a hablar, de un lado de paz a nivel político y de otro lado, paz a nivel social.

Esto se ha convertido en un punto fundamental ya que

(...) es imposible entender los procesos económicos y sociales de Colombia desde comienzos de los años noventa sin entender la dialéctica entre los esfuerzos de modernización económica, representados en la apertura, y la búsqueda de un nuevo pacto social, cuya materialización más importante fue la Constitución de 1991 (Ocampo, 2004. 17.)

Como lo señala José Antonio Ocampo, la tensión "entre una nueva Constitución que pedía más Estado como mecanismo para superar la crisis institucional, y un plan de desarrollo que diagnostica la necesidad de menos Estado, es el eje de la reforma del Estado en Colombia" (Ocampo, 2004, 17).

Este tema sería entonces, el eje principal para la gobernabilidad de nuestro país.

Desde esta postura, la Constitución se enfoca en tres momentos que caracterizan su estructura y finalidad, en un primer momento propone una nueva legislación centrada en el reconocimiento de los derechos, el funcionamiento institucional y el modelo de desarrollo, en segundo momento se reconoce derechos específicos a las comunidades étnicas afrodescendientes e indígenas, y en tercer momento otorga un lugar particular a los actores sociales y su participación en la regulación política, motivo por el cual esta constitución ahora planteada permitiría ver mayores niveles de gobernanza, pues existiría un fortalecimiento del Estado donde hay apertura a la sociedad en la participación de asuntos públicos.

Sin lugar a duda, las reformas institucionales, el reconocimiento de los grupos étnicos y la participación de actores no estatales en los asuntos públicos que encarna la Constitución representa un esfuerzo notable para la ampliación de la democracia. Sin embargo, hay que reconocer que la democracia no sólo descansa en textos legislativos sino en una interacción compleja de actores sociales y políticos cuyo papel es fundamental tomar en cuenta (Launay y González, 2010, 141).

No se trata, entonces, solamente de impulsar el fortalecimiento del Estado de derecho y de las instituciones políticas, sino también de un esfuerzo que vaya más allá de los aspectos legales que regulan las relaciones entre los ciudadanos y el Estado para tomar en cuenta las formas como los pobladores y las comunidades entienden, utilizan y valoran la ley y sus instituciones (Comisión de Seguridad Humana, 2003, 76), es decir, que uno de los puntos de atención del Estado sería la preocupación por aquellos aspectos sociológicos e históricos que caracterizan a cada nación, en lugar de ofertar marcos normativos y fórmulas estandarizadas de ayuda y cooperación internacional, según los modelos abstraídos de las experiencias de sus propios países.

Pensar la gobernanza en Colombia significaría establecer un equilibrio entre lo global y lo local, y adoptar una mayor flexibilidad sobres sus supuestos teóricos para poder indagar las causas que subyacen a los problemas e identificar las vicisitudes concretas a las que se enfrentan las políticas, programas y normas pensadas para crear las condiciones que permiten alcanzar el desarrollo, la superación del conflicto y la gobernabilidad democrática (Launay y González, 2010, 147).

A pesar de que los intentos por hacer un cambio político a partir de la Constitución del 91, fueron propuestos en gran escala y con las mejores intenciones, se percibieron resultados inesperados, ya que: 
La gobernanza y la gobernabilidad fue la manera como la descentralización administrativa y fiscal, que se suponía el instrumento ideal para acercar el Estado a las comunidades y pacificar las sociedad colombiana, terminó contribuyendo exactamente al resultado contrario: facilitar la inserción de grupo armados ilegales, guerrillas y paramilitares efectivamente en las instituciones de la vida política local, pero sin necesidad de desmovilizarse previamente, tal y como lo devela el proceso judicial de la parapolítica. Se pensaba ingenuamente que bastaban medidas técnicas y descentralizadoras para mejorar las relaciones entre lo local, lo regional y lo nacional, por no tener en cuenta las complejidades concretas de esa interacción. (Launay y González, 2010, 199).

Sin embargo, no se puede desconocer que también hubo efectos positivos a partir de esta Constitución, ya que se da una consolidación concreta en cuanto a la creación de nuevos municipios y la formación de nuevos liderazgos en aquellos lugares marginados del territorio, lo que permitió un dinamismo en la vida política local al estimular, de un lado, la creación de ejercicios de planeación colectiva y de otro la opinión pública capaz de exigir transparencia en las decisiones públicas.

En este sentido, se puede entender que una sana política de gobernanza, es aquella que tiene en cuenta las particularidades regionales y locales y asume de manera acertada su realidad, todo con miras a prever y neutralizar aquellos problemas que se pueden presentar en el dinamismo, que es propio de la política.

\section{Conclusiones}

La gobernabilidad asume la idea de que aquellas instituciones propias del Estado, y que al parecer, se encuentran bien estructuradas, son las que permiten escuchar y de igual manera responder de manera eficiente a las demandas de la sociedad civil; es aquí donde se habla de buena gobernabilidad, porque se presenta una respuesta inmediata de manera gubernamental, frente a las situaciones que afectan a su misma sociedad. En cambio, la gobernanza se entiende como un estilo de gobernar, que tiende a un mejor desarrollo económico, social e institucional, en donde se pueda establecer una verdadera relación entre el Estado, la sociedad y su economía, en donde se amplíe los niveles de desarrollo. Estos conceptos asumen la sociedad como una realidad que debe estar presente y ser tenida en cuenta por las actividades, funciones y proyectos propios de un Estado, ya que la sociedad misma es el sentido de ser de un país.

En este mismo sentido, se pudo ver una situación más donde la sociedad se encuentra involucrada, no como el fin, sino como el medio; aquellos momentos de conflicto, en donde la presencia del Estado es mínima, y la de los actores armados es amplia, situación en la cual, la sociedad pone sus demandas o necesidades inmediatas en manos de aquellos grupos, pues ven en ellos un medio para obtener lo que siempre han pedido y no han podido lograr. Al parecer los actores armados producen como dice Launay y González, una nueva forma gobernanza/ gobernabilidad que vaya más allá de las formas institucionales que busca explicar las relaciones sociales y políticas en las sociedades $(2010,101)$.

Sin embargo, en la realidad colombiana se contó con la reforma constitucional de 1991, con el fin de recuperar la legitimidad del régimen político y mejorar las prácticas de gobernanza. Una nueva propuesta constitucional, que buscaba establecer una relación más estable entre gobernantes y gobernados, en el momento mismo en que consagraba los derechos y el carácter pluralista de nación en aspectos religiosos, étnicos, culturales, con un aspecto adicional como era el 
de los mecanismos de participación, ya que se quería lograr un equilibrio entre las demandas sociales y la capacidad de atención estatal.

\section{Referencias}

Diccionario de la Real Academia Española: http:// buscon.rae.es/drael/ SrvltConsulta? TIPO BUS3\&LEMA=gobernanza

González, F. Bolívar, I. y Vásquez, T. (2003). Violencia política en Colombia. De la nación fragmentada a la construcción del Estado. Bogotá: CINEP.

Kingma, K. (2001). Demobilisation and reintegration of ex-combatants in post-war and transition countries. Trends and challenges of external support. Alemania: Bonn International Center for Conviction BICC

Kooiman, J. (2003). "Governance and Governability". En J. Kooiman. Governing as Governance. London: Sage Publications.

Koth, M. (2005). To end a war: demobilisation and reintegration of paramilitaries in Colombia. Alemania: Bonn International Center for Conviction BICC.
Launay, G. y González, F. (2010). Gobernanza y conflicto en Colombia. Interacción entre Gobernantes y Gobernados. Bogotá. Edit. Pontificia Universidad Javeriana. $225 \mathrm{p}$

Ocampo, J. (2004). Entre las reformas y el conflicto, economía y política en Colombia. Colombia: Norma.

Pauwels, N. (2000). War force to work force: global perspectives on demobilization and reintegration. Bonn International Center for Conversion. Nomos Verlagsgesellschaft, Baden-Baden.

Rettberg, A. (2002). Preparar el futuro: conflicto y posconflicto en Colombia. Colombia: Libros cambio, Universidad de los Andes, Fundación Ideas para la Paz, ALFAOMEGA.

Santana, P. (1990). Crisis institucional y legitimidad política en Colombia. En Revista Foro. Núm. 12 (Junio). Bogotá.

Ungar, E. (1997). "Introducción". En: Elizabeth Ungar (edit). Gobernabilidad en Colombia retos y desafíos. Bogotá: Departamento de Ciencia Política - Universidad de los Andes. 